\title{
Krabbe Disease
}

National Cancer Institute

\section{Source}

National Cancer Institute. Krabbe Disease. NCI Thesaurus. Code C61254.

A rare inherited neurodegenerative disorder that belongs to the group of

leukodystrophies. It is characterized by myelin destruction, gliosis in the brain, and the presence of multinucleated globoid cells. Signs and symptoms include irritability, mental and motor developmental disturbances, muscle weakness, seizures, blindness, and deafness. 